\title{
Cartografías colectivas como espacios de reflexión visual: territorios, experiencias y contextos
}

\section{Collective cartographies as spaces for visual reflection: territories, experiences and contexts}

TIPO DE TRABAJO: Comunicación virtual.

PALABRAS CLAVE

Cartografía, obra artística, aprendizaje formal, formación docente, diversidad.

KEYWORDS

Cartography, artistic work, formal learning, teacher training, diversity.

RESUMEN

La presente comunicación, plantea las posibilidades de la creación de cartografías de autoría colectiva como espacios reflexivos.

Las cartografías visuales representan un recurso versátil y contextualizado al trabajo reflexivo que se puede hacer con diversos grupos humanos, en este punto se puede comprender que el trabajo cartográfico además de representar la construcción de una obra artístico visual puede ser una reflexión colectiva que involucra el territorio, las experiencias personales y los contextos diversos. De esta forma la experiencia de cartografía visual reflexiva es también una cartografía social y participativa (Barragán, 2016).

En este caso se presentarán dos experiencias en contextos de aprendizaje formal tanto escolar como universitario: el primero sobre reconocimiento de autoridad femenina en los procesos de aprendizaje en una escuela pública para mujeres y el segundo sobre la configuración de identidad docente en el caso de estudiantes de pedagogía LGBTI+, ambos en Santiago de Chile.

De esta manera las cartografías se transformaron en un re-conocimiento e intervención del territorio desde su apropiación llevando a cabo bordados colectivos en tela como botones e hilos donde iban marcando hitos relativos a los temas propuestos al principio de la actividad. En el caso de la escuela fueron trazando lugares de aprendizaje entre mujeres, simbolizando a estas con botones, las que interactuaban según sus lugares de residencia en el mapa de la ciudad, lo que evidencia la ocupación de los barrios y los lugares donde existe interacción en femenino. Mientras que por otro lado en la experiencia con el estudiantado LGBTI+, las interacciones en el territorio estuvieron dadas por sus propias trayectorias de vida desde los conflictos con el yo, reconocerse parte de la diversidad sexual y sobre todo los lugares que representan el miedo.

\section{ABSTRACT}

This article raises the possibilities of collaborative cartographies creation as spaces for reflection.

Visual cartographies represent a versatile and contextualized resource to the reflexive work that can be done with different human groups. At this point it can be understood that the cartographic work besides representing the construction of a visual artistic work can be a collective reflection that involves territory, personal experiences and diverse contexts. In this way, the experience of reflexive visual cartography is also a social and participative cartography (Barragán, 2016).

In this case, two experiences will be presented in formal learning contexts, both at the school and university level: the first on the recognition of female authority in the learning processes in a public school for women and the second on the configuration of teaching identity in the case of LGBTI+ students of pedagogy, both in Santiago de Chile. 
In this way, the cartographies were transformed into a re-knowledge and intervention of the territory from its appropriation, carrying out collective embroidery on canvas with buttons and threads that symbolize milestones related to the themes proposed at the beginning of the activity. In the case of the school, they traced places of learning among women, symbolizing these with buttons, which interacted according to their places of residence on the map of the city, which shows the students territorial distribution and those places where female interactions occur. While on the other hand in the experience with the LGBTI + student, the interactions in the territory were given by their own life trajectories from the conflicts with the self, recognizing as part of the sexual diversity and especially the places that cause them fear.

\section{INTRODUCCIÓN}

El trabajo cartográfico presenta innumerables ventajas al momento de presentarlo como la construcción de obra colectiva donde su propia construcción se plantea como reflexiva y dialógica en un contexto determinado. En este artículo se presentarán dos casos de construcción cartográfica colectiva con énfasis en la reflexión visual que se configura a partir del propio diseño cartográfico que involucra la conciencia del territorio, la propia ubicación en el espacio y el lugar de otros/as, lo que da pie a la discusión de infinidad de temas que están vinculados a estos conceptos y a las propias experiencias de los/as participantes de la elaboración de la obra.

\section{MARCO TEÓRICO}

Para Rolnik (2006) Cartografiar es un acto que no sólo se preocupa de la estética de los mapas realizados, sino que también se modifica según las transformaciones del paisaje y sus sociedades. Se plantea entonces como la construcción de mapas de un territorio determinado (real o imaginario) y que en este caso se configura no sólo como la representación de un lugar, sino también como la construcción de una obra y como el proceso para llevar a cabo reflexiones visuales a partir esta.

\section{- Las cartografías como espacio de reflexión visual}

Los recursos cartográficos tienen la particularidad de presentar un territorio, sea cual sea e invitar a observar, reflexionar y vincular temáticas diversas con esos territorios. A partir de aquí, se puede realizar obras artísticas que sean el propio mapa y que visibilicen las reflexiones realizadas a partir del espacio mapeado a través de los lenguajes artísticos.

El ser humano ha intentado representar el mundo circundante por medio de diversas formas creativas: narraciones, arte, escritura, fotografía, entre otras tantas; en esta amalgama de posibilidades, los mapas suelen ser una producción humana en la que se intenta comprender lo circundante ya sea en relación con la geografía o con lo que está más allá de lo terreno. (Barragán, 2015, p.248).

En este sentido, los mapas se transforman en la organización visual de un tema tratado y que se constituye como una reflexión visual que da cuenta de las propias experiencias y contextos de quienes participan en el desarrollo de la obra, transformándose en "espejos, representaciones gráficas de algunos aspectos del mundo real" (Harley, 2005, p.73). De esta manera la colectividad participante otorga espacio a las propia biografía para desarrollar la obra colectivamente, encontrando lugar en este mapeo.

\section{- Posibilidades de reflexión a partir de la ubicación en el territorio: dos casos}

Comprendiendo entonces el rol de la obra cartográfica asociada a un proceso reflexivo, es necesario plantear las características de ese proceso reflexivo que deriva en la construcción de una obra artística. De esta manera, uno de los puntos más relevantes para llevar a cabo una reflexión significativa durante el mapeo colectivo es precisamente la importancia de la propia experiencia y los contextos con los que me vinculo al territorio. Emerge el concepto de Partir de sí (Piussi, 2000) como "una forma de hablar o construir conocimientos sobre algo pero partiendo de mis propias experiencias en contextos determinados que hacen que esa experiencia sea particular" (Montenegro, 2017, p.413). Vinculado a esto se puede plantear también la importancia del contexto y como en este caso, se relaciona con el territorio desde el cual estoy llevando a cabo la reflexión "es construir una subjetividad, construir figuras del YO y así partiendo del yo se llega al sí mismo en relación con el otro" (Viollet, 2005, p.27), propiciando además una relación con quienes estoy llevando a cabo la cartografía, dando lugar a los espacios de escucha, respeto y valoración de las experiencias de otros/as. Es por lo anterior que las cartografías presentadas en esta comunicación plantean dos actividades reflexivas en torno precisamente a las experiencias cotidianas: por un lado el caso 1, que busca reconocimiento de autoridad femenina vinculada a los procesos de aprendizaje diario donde se propicia "la experiencia personal de fascinación por la maravilla de aprender en los encuentros diarios en las aulas" (Rivera, 2012, p.89) a través de una elaboración de obra.

Otro aspecto importante es el referido al caso 2, Formación del profesorado LGBTI+ considerando el futuro profesional de quienes participan en la experiencia cartográfica y las inquietudes del incierto futuro profesional comprendiendo que "La 
escuela es un lugar privilegiado de sociabilización, en ella toman lugar diferentes procesos de identificación, de subjetivación y sobre todo de interrelación; pero también de violencia, exclusión y discriminación" (Rojas y otros, 2018, p.14), es por esto que se hace necesario reflexionar sobre el rol del profesorado de las diversidades sexuales para la formación del estudiantado en libertad pero también valorando la diferencia e individualidad de todas las personas que configuran la comunidad educativa.

\section{METODOLOGÍA}

Ambas experiencias fueron trabajadas en espacios de educación formal. El primer caso en una escuela secundaria tradicional y emblemática dirigida a la formación de mujeres con alta exigencia académica. Se trabaja en la clase de artes visuales con el objetivo de Crear una obra artística colectiva que refleje las experiencias personales en torno a una problemática social. La segunda experiencia en el contexto de un taller extra-programático en la Universidad Católica Silva Henríquez.

Ambos parten de una metodología de trabajo teórico-práctico en tanto los temas tratados. En el primer caso se trabajaron los temas referidos a autoridad femenina (Rivera, 2012), aprendizaje considerando el trato amoroso y los afectos (Montoya, 2008) y el orden simbólico de la madre (Muraro, 1995). Mientras que en el segundo caso se trabajaron temáticas referidas a diversidad sexual (Tomé, y Rambla, 2001; SERNAM, 2009) y formación docente, esto vinculado a diversidad sexual (Barrientos, Andrade y Montenegro, 2018). Según lo anterior, el trabajo teórico es la base de las sesiones reflexivo-visuales que se llevan a cabo mientras se realiza la construcción colectiva de las cartografías sobre tela.

Luego de llevar a cabo un trabajo-teórico práctico se realiza una reflexión final que toma forma gracias a la investigación biográfico narrativa comprendiendo esta como la presentación de la "experiencia concreta humana como una descripción de las intenciones, mediante una secuencia de eventos en tiempos y lugares, en donde los relatos biográfico-narrativos son los medios privilegiados de conocimiento e investigación" (Bolívar, 2002, p.9) propiciando un círculo de reflexión donde las/los participantes comparten las ideas más importantes de la experiencia vivida.

De esta manera se da relevancia a la vinculación de la obra con las propias experiencias personales, siendo estas últimas precisamente lo que da forma definitiva a la obra según las rutas, espacios y comunas habitados por quienes participaron de los casos. Por otra parte, se podría plantear un enlace con la investigación basada en las artes dada la construcción colectiva de la obra, dando vida a una reflexión que se desarrolla mientras se realiza la propia obra, transformándose en una cartografía colectiva como reflexión visual, (como se menciona al principio de la comunicación). Esta última propuesta está vinculada con la necesidad del propio estudiantado de investigar a partir de su práctica artística y la mía como docente de la asignatura, es por ello que se propone una metodología de trabajo en concordancia con metodologías de investigación.

\section{DESARROLLO}

\section{Caso 1.- La cartografía de los aprendizajes amorosos: reconocimiento de autoridad femenina en los procesos de aprendizaje}

En el contexto de la asignatura de artes visuales en un colegio público, tradicional y de alta exigencia de mujeres de Santiago de Chile se lleva a cabo una actividad cartográfica que persigue relevar la importancia de las mujeres en los procesos de aprendizaje informal de otras mujeres jóvenes (estudiantes de secundaria entre 15 y 16 años).

En conjunto con las estudiantes nos pusimos a pensar en formas de volver a tener cerca los afectos en la educación, pensar cómo en las discusiones sociales, podíamos reconocer a las mujeres, a nuestras maestras, a las que nos enseñaron desde el amor y el afecto ¿Cómo podíamos reconocer el aprendizaje en femenino? Comenzamos con las estudiantes de segundo año de secundaria a discutir sobre estas inquietudes y pensamos en conjunto hacer una cartografía de los aprendizajes amorosos. Se hizo el mapa de Santiago y las estudiantes pusieron botones donde vivieran esas mujeres que nos habían enseñado con amor y luego unieron esos botones con hilos rojos a los botones que las representaban a ellas mismas.

\section{Articulación de las sesiones:}

En primer lugar dibujaron el mapa en la tela para luego bordar en estilo libre los márgenes comunales y los nombres de las mismas. En paralelo a esto las estudiantes conversaban sobre las mujeres que representarían mediante botones de colores. Aquí pegaban los botones a la tela para representar a las mujeres y otro que las representaba a ellas, para luego unirlos entre sí. Luego de esto y uniendo los botones las estudiantes expusieron el trabajo a la comunidad educativa donde compartieron verbalmente la relevancia de la construcción de la obra, compartieron los significados de esas mujeres representadas por botones y cuánto de ellas pervivía aún en sus vidas. 

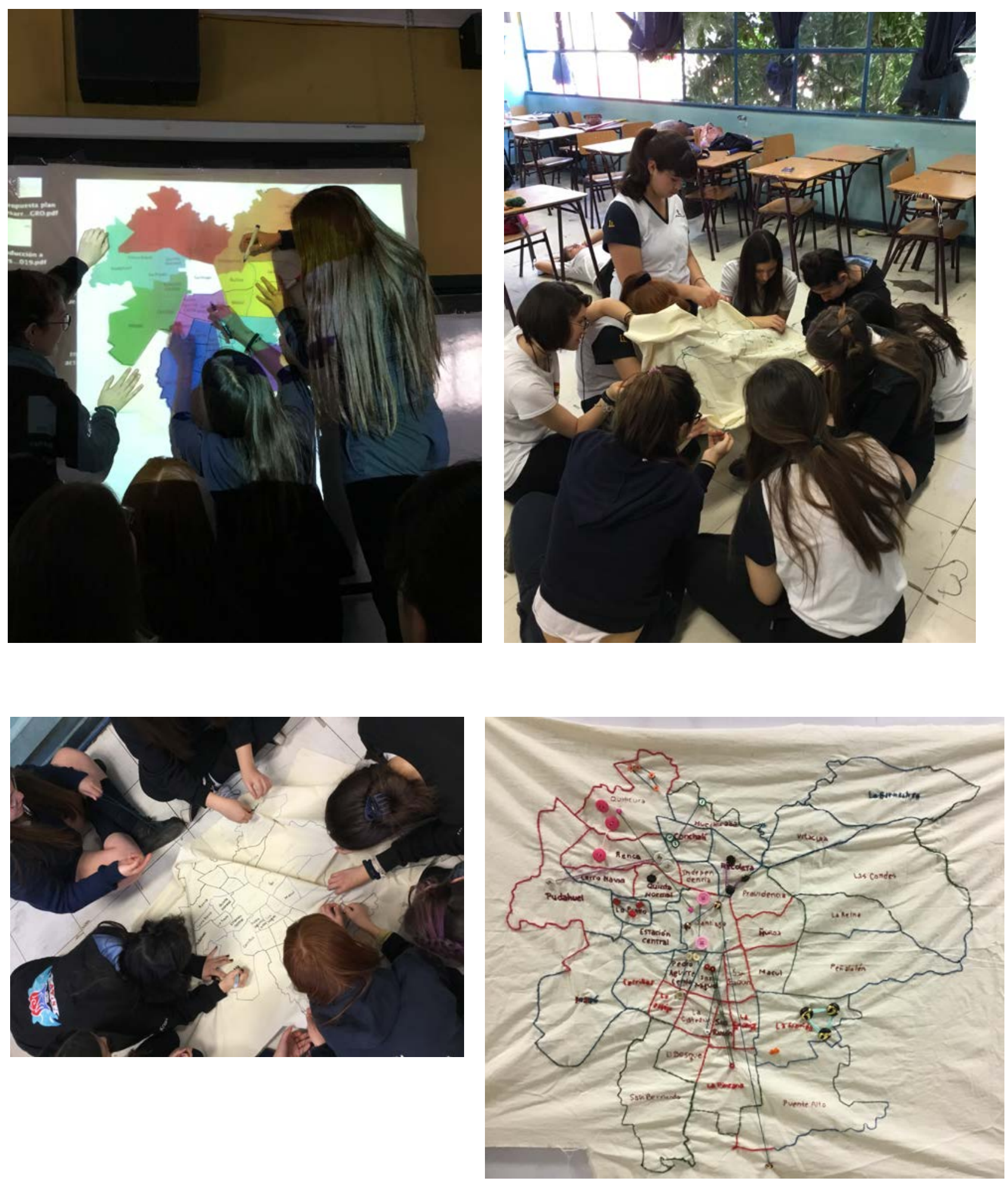

Figura 1: Fotografías caso 1 (registro propio) 


\section{Caso 2.- La cartografía del miedo: los territorios y la configuración de identidad docente en el caso de estudiantes de pedagogía} LGBTI+

La actividad cartográfica se llevó a cabo con estudiantes LGBTI+ de carreras de pedagogía en Artes visuales, Música y Matemáticas, fueron tres estudiantes en total, de los cuales dos eran hombres homosexuales y un hombre trans. Con estos tres estudiantes se llevan a cabo tres sesiones de mapeo en dependencias de la Universidad Católica Silva Henríquez. En el caso de este grupo la actividad emerge como inquietud de ellos mismos quienes plantean la necesidad de reflexionar sobre su pertenencia a minorías sexuales en proceso de formación docente, donde declaran que no han sido preparados o no han tenido actividades donde se visibilice el rol de docentes de minorías sexuales y que esto a su vez les produce mucha ansiedad y sobre todo miedo a salir al mundo laboral en un contexto escolar que reproduce brechas de género, discriminación y estereotipos de manera explícita a día de hoy (Contreras, 2004; Fundación Todo Mejora, 2016; MINEDUC, 2015-2018).

\section{Articulación de las sesiones:}

Los encuentros son divididos en tres sesiones de las cuales la primera tiene la intención de abrir el diálogo en torno a sus recorridos biográficos desde el dentro del closet, salir del closet y formarse como docentes en este último lugar simbólico. Posterior a esto, los estudiantes identificaron en el mapa los lugares que pudieran representar algún miedo social como individuos y como futuros docentes en contexto escolar. Luego de dibujar el mapa en la tela, fueron marcando con diversos elementos (botones, figuras de plástico, plumas entre otros objetos) los lugares mencionados, intentando que la carga simbólica del lugar previamente conversada, tuviera relación con el objeto que lo representa.

Dentro de los temas más relevantes visibilizados en las sesiones se presenta la idea de la homosexualidad como un discurso político (estudiante de Artes visuales), esto en la lógica de que como futuros docentes pueden transformarse en una persona/lugar seguro para sus estudiantes pertenecientes a las diversidades sexuales, esto considerando los altos niveles de bullying homofóbico presentes en Chile, donde el 38.3\% de estudiantes que respondieron la Encuesta de la Fundación Todo Mejora (2016) declara algún tipo de hostigamiento por Expresión de género. Esta idea contrasta con lo declarado por el estudiante transgénero quien plantea su profundo miedo a las agresiones físicas en contexto social, lo que le hace pensar que evitaría hablar sobre su identidad de género en un futuro contexto profesional docente dado los constantes ataques que ha recibido a lo largo de su vida. Mientras que el estudiante de la carrera de matemáticas plantea que no es un tema para hablarlo públicamente y le parecería innecesario visibilizarlo con sus futuros estudiantes de escuela.
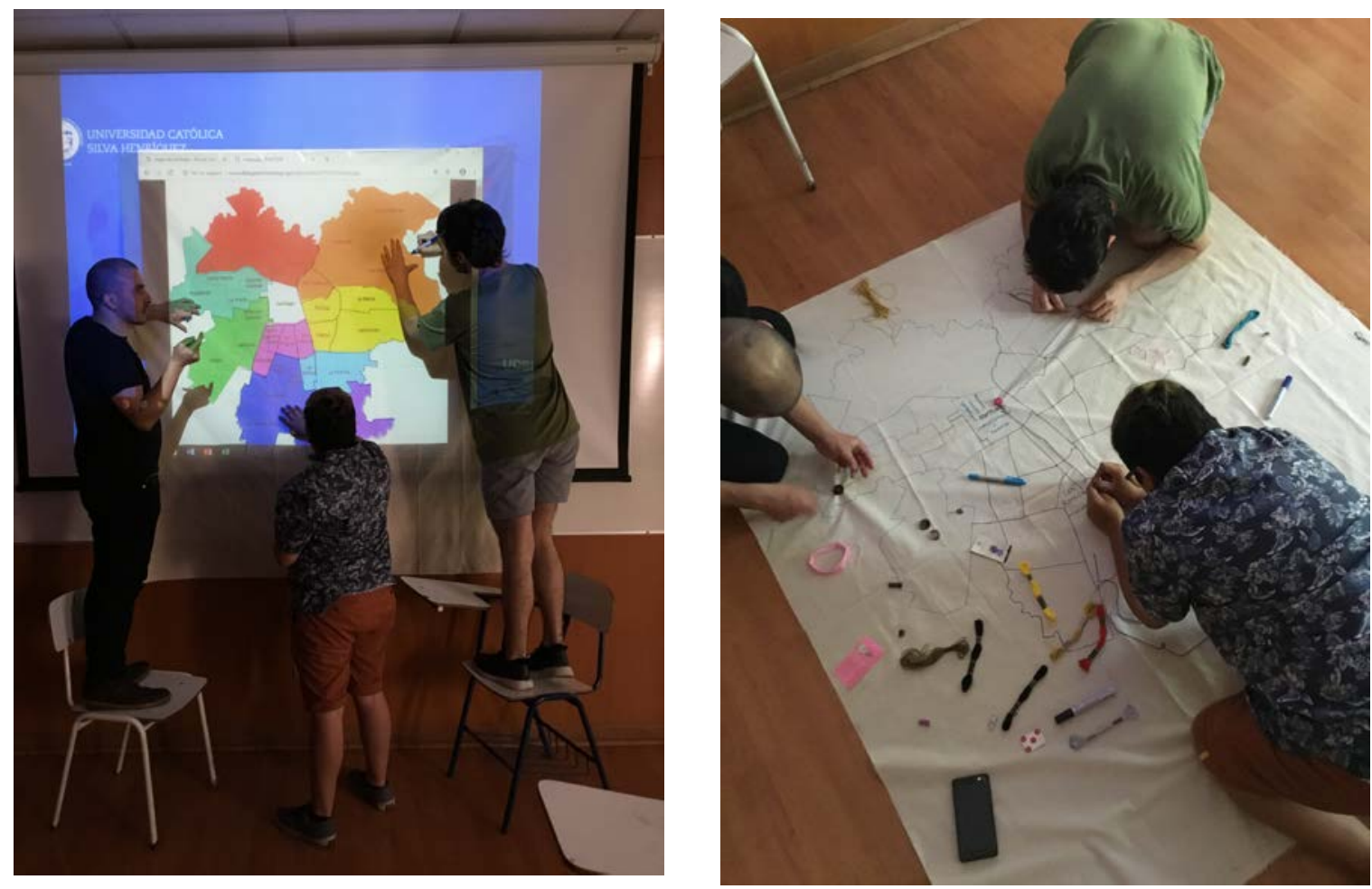

Figura 2: Fotografías caso 2 (registro propio) 


\section{Materialidad y recursos}

Teniendo una distancia temporal entre el primer caso y el segundo, se muestra como ejemplo material la cartografía de las estudiantes de secundaria a el estudiantado en formación docente. Al mostrar la primera experiencia, los docentes en formación deciden replicar el mapa y vincular las experiencias utilizando el mismo soporte de tela e hilos para bordar. Estos últimos agregan accesorios que en Chile son popularmente vinculados con personas de las minorías sexuales como plumas, brillos y lentejuelas como una forma de ironía y de respuesta a los estereotipos de género presentes en el país.

\section{CONCLUSIONES}

En el caso 1, la ubicación en el territorio permitió que las reflexiones fueran vinculadas a los lugares con mayor cantidad de estudiantes en cada comuna, lo que les hacia pensar en sus lugares de origen, los vacíos mapeales y la reflexión sobre los lugares de procedencia vinculados a clase social y donde se ubican según esta, comprendiendo el rol de la escuela como formadora de mujeres de clase social baja y media y de cómo este espacio de aprendizaje formal se configura como una manera de empoderar a mujeres de los estratos sociales antes mencionados.

En el caso de la cartografía del miedo (caso 2) se vincularon los lugares de la ciudad con experiencias traumáticas de violencia y miedo asociadas a su pertenencia al colectivo LGBTI+ sin embargo, en el caso de uno de los participantes, residente en un sector de clase alta de la ciudad se pudo dialogar en torno a su ausencia de experiencias traumáticas callejeras asociadas a su homosexualidad lo que hace pensar que las agresiones no son solo al colectivo LGBTI+ sino que además se suma la condición de vulnerabilidad o pobreza de los sectores identificados en el mapa como peligrosos, a evitar o donde recibieron agresiones físicas y verbales.

Este tipo de obras colectivas pueden ser realizables en diversos contextos, permitiendo el desarrollo reflexivo a partir de múltiples temas vinculados a los territorios habitados por quienes participen de las experiencias, propiciando el diálogo y el trabajo colaborativo en la construcción de la obra de autoría múltiple, respetando la vinculación de los lugares con las experiencias personales, singulares e irrepetibles, dando espacio al auto-relato que deriva en la construcción de una obra artística a partir de las historias de vida de quienes participan.

\section{FUENTES REFERENCIALES}

Barragán, D. (2015). Cartografía social pedagógica: entre teoría y metodología. Revista colombiana de educación, 70, $247-285$.

Barrientos, P., Andrade, D. y Montengro, C. (2018). La formación docente en género y diversidad sexual: Tareas pendientes, Cuaderno de Educación, 81, 1-13.

Bolívar, A. (2002). “¿De nobis ipsis silemus?”: Epistemología de la investigación biográfico-narrativa en educación. Revista Electrónica de Investigación Educativa, 4 (1). Consultado el día de mes de año en: http://redie.uabc.uabc.mx/vol4no1/contenido-bolivar.html

Centro de Investigación y Desarrollo de la Educación (cide), Universidad Alberto Hurtado (2018). Narrativas, prácticas y experiencias en torno a la identidad Igbti en contextos educativos: Informe final. Encargado por mineduc y unesco-orealc, Chile. Rojas, M., Stefoni, C., Fernández, M., Salinas, P., Valdebenito, M. \& Astudillo, P.

Contreras, M. (2004). Educación y Género. Un desafío de la organización magisterial. Santiago: SERNAM/Colegio de Profesores.

Harley, J. B. (2005). La nueva naturaleza de los mapas. Ensayos sobre la historia de la cartografía. México: fce.

Ministerio de Educación. (2015). Educación para la igualdad de género 2015-2018. Recuperado de https://media.mineduc.cl/wpcontent/uploads/sites/28/2017/07/Educaci\%C3\%B3n-para-la-lgualdad-de-G\%C3\%A9nero-2015-2018.pdf

Montenegro, C. (2017). Cultura visual y pedagogías feministas: repensando los espacios educativos. Publicación del Congreso Internacional Arte, Ilustración y Cultura Visual en Educación Infantil y Primaria, 4a edición: Mediación crítica dentro y fuera de la escuela, (pp. 347- 353). Universidad Pública de Navarra, San Sebastián.

Muraro, L. (1995). El orden simbólico de la madre. Madrid: Horas y horas 
Piussi, A. (2000). Partir de sí: necesidad y deseo. DUODA: estudis de la diferència sexual, 19, 107-114.

Rivera, M. (2012). El amor es el signo. Educar como educan las madres. Madrid: Sabina.

Rolnik, S. (2006). Cartografía sentimental. Porto Alegre: Editora da UFRGS.

Servicio Nacional de la Mujer. (2009). Documento de Trabajo No 117, Análisis de Género en el Aula. Recuperado de https://hivhealthclearinghouse.unesco.org/sites/default/files/resources/santiago rec 20090318 121034.pdf

Fundación Todo Mejora (2016). Encuesta nacional de clima escolar. Disponible en https://todo-mejora.org/wpcontent/uploads/2016/08/Encuesta-de-Clima-Escolar-2016-Fun-dacion-TODO-MEJORA.pdf

Tomé, A. y Rambla, X. (2001). Contra el sexismo: coeducación y democracia en la escuela. Barcelona: Síntesis.

Viollet, C. (2005). Pequeña cosmogonía de escritos autobiográficos. Cuadernos de crítica de la cultura, (69), 23-29. 